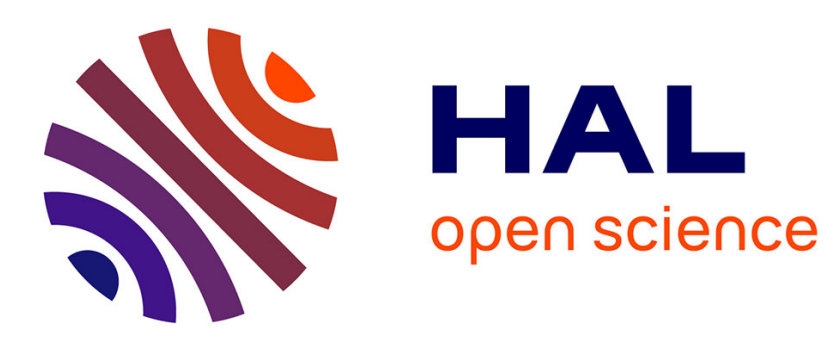

\title{
Poisson et dragon: symboles du véhicule entre l'ici-bas et l'au-delà
}

Daeyeol Kim

\section{To cite this version:}

Daeyeol Kim. Poisson et dragon: symboles du véhicule entre l'ici-bas et l'au-delà. Cahiers d'ExtrêmeAsie, 2004, 14, pp.269-290. halshs-00349926

\section{HAL Id: halshs-00349926 https://shs.hal.science/halshs-00349926}

Submitted on 30 Dec 2015

HAL is a multi-disciplinary open access archive for the deposit and dissemination of scientific research documents, whether they are published or not. The documents may come from teaching and research institutions in France or abroad, or from public or private research centers.
L'archive ouverte pluridisciplinaire HAL, est destinée au dépôt et à la diffusion de documents scientifiques de niveau recherche, publiés ou non, émanant des établissements d'enseignement et de recherche français ou étrangers, des laboratoires publics ou privés. 


\title{
Poisson et dragon : symboles du véhicule entre l'ici-bas et l'au-delà
}

\author{
KIM Daeyeol
}

Taoist texts dating from the fourth to sixth centuries mention the throwing of the figurine of a fish with one of man or a message in a river as a sign of the oath taken at the moment of the transmission of a secret document or recipe. The aim of this article is to explain this ritual act.

In certain texts and scenes from tombs in early China, the fish appears as a vehicle of immortals or spirits. In medieval times, the form of the fish was used for tallies involving communication between two parties distant from each other. With regard to ritual context and morphology, the "throwing of the fish" is analogous to the "throwing of the dragon" studied by Chavannes. The dragon in this rite represented the "postal service" for communicating with the gods, and some scholars consider this practice to derive from the dispatch of messages to the Officers of Heaven, Earth and Water in early Heavenly Master Taoism. The throwing of fish may therefore be interpreted as a ritual act designed to send the message of the initiate to the world of the immortals.

Cet article tente d'expliquer le geste rituel qui consiste à jeter dans un cours d'eau une statuette de poisson en or avec une autre en forme d'homme, lors du rite de transmission des recettes alchimiques en Chine ancienne. On le trouve dans certains textes alchimiques des origines, datant des Han de l'Est (25-220) ou des Six Dynasties (222-589). Une interprétation de cette pratique s'impose, le rôle du poisson n'étant pas explicité dans les textes.

Ce «jet du poisson » s'inscrit, nous semble-t-il, dans le même contexte symbolico-religieux que le « jet du dragon». Ce dernier, appelé toulong 投 龍, est connu par une étude d'Edouard Chavannes (1919) et par d'autres travaux récents. Il s'agit d'un rite taoïste consistant à jeter dans les montagnes ou cours d'eau célèbres une ou des figurines de dragon en or, avec ou sans autres objets comme des plaques de jade ${ }^{1}$. Le jet du dragon, mentionné dans certains textes taoïstes dès le $5^{\mathrm{e}}$ siècle après J.-C. et pratiqué jusqu'à l'époque des Ming, était effectué pour faire parvenir hommage, vœu, prière, etc. auprès des divinités célestes.

Cet article a bénéficié des critiques et remarques judicieuses de Michèle Pirazzoli et de John Lagerwey. Qu'ils trouvent ici l'expression de ma reconnaissance. Je remercie également Laurence Denès pour sa relecture attentive du texte. Il va de soi que je suis seul responsable des erreurs de toutes sortes.

${ }^{1}$ Dans les rituels de jet du dragon, il arrive que ce dernier soit associé à des objets en jade : voir les études citées ci-après. De même, le jet du poisson est aussi associé à l'anneau de jade : voir Zaozhuangshi wenwu guanli weiyuanhui bangongshi et Zaozhuangshi bowuguan 1997, p. 42. 
Les chercheurs ont mis le jet du dragon en rapport avec l'ancien rite consistant pour le Fils du Ciel en l'offrande d'un yubi 玉璧, anneau de jade, aux divinités, et avec le culte des sanguan 三官, Trois Officiers, du mouvement taoïste des Cinq boisseaux de riz. Le sanguan shoushu 三官手 書 et le jet du dragon sont ainsi placés ensemble dans la continuité de l'évolution des anciens cultes rendus aux esprits de la nature, tels ceux du Ciel, des montagnes et des fleuves : le wang 望, sacrifice offert aux esprits des montagnes et des cours d'eau que l'on trouve, par exemple, dans le Zuozhuan 左傳; le $\mathrm{Zu}$ Chu wen 詛楚文, où le roi de Qin dénonce le royaume de Chu auprès des divinités de la nature ; les sacrifices feng et shan 封禪, offerts respectivement au Ciel et à la Terre par les empereurs ${ }^{2}$.

Comme le rite du jet du poisson revêt un caractère sacrificiel lié à l'eau, nous pouvons l'associer à la même tradition cultuelle. Cependant, le jet du poisson et le jet du dragon se singularisent par l'usage des symboles qui accompagnent l'offrande ou le message envoyés aux divinités. Ils relèvent très probablement du même système de signification. Il est aussi possible que le jet du poisson ait coexisté avec le jet du dragon, ou qu'il l'ait précédé aux premiers siècles de notre ère ${ }^{3}$. Quelques textes taoïstes et certaines représentations symboliques du poisson incitent à le penser. Aussi proposons-nous une étude centrée sur la signification du symbole du poisson et sur le contexte rituel du jet du poisson et du jet du dragon. Deux axes de recherche sont donc privilégiés : la fonction symbolique du poisson dans les relations entre deux mondes séparés et les points communs, du point du vue $\mathrm{du}$ rituel, entre les gestes pratiqués autour du poisson et autour du dragon. Concernant la seconde question, il est notoire que, sur le plan symbolique et mythique, le dragon et le poisson sont intimement liés l'un à l'autre. Ainsi, par exemple, ils se transforment mutuellement l'un en l'autre ; ils servent tous deux de montures aux Immortels ${ }^{4}$. Dans cet article nous limitons donc le sujet à la question des relations qui lient le poisson et le dragon avec le monde des Immortels ou des divinités et avec la transmission des écrits secrets.

Commençons par présenter le jet du poisson. L'itinéraire des alchimistes, tel que le décrivent le Baopuzi neipian ou Chapitres Intérieurs de Ge Hong (283-343) et les premiers textes de l'alchimie taoïste, commence par la

2 Tai Jingnong 1975, p. 323 ; Rao Zongyi 1989, p. 109 ; Wang Yucheng 1994, p. 152 ; Zhang Zehong 1996, p. 29 ; Zhou Xibo 1999, p. 92 ; Li Ling 2000, p. 457-460 ; Liu Zhaorui 2001.

${ }^{3}$ Liu Zhaorui $(2001,493)$ pense que le jet du dragon prend forme au cours de la période qui va des Jin de l'Est (317-420) aux Dynasties du Nord et du Sud (420-589).

${ }^{4}$ En ce qui concerne l'affinité entre le poisson et le dragon, voir par exemple Granet 1926, p. 261, 326, 344-350, 519 ; Stein 1947, p. 265 s., 275-278; Kaltenmark 1948, p. 21 ; Kaltenmark 1953, p. 106 ; Diény 1987, p. $139-156$ et 173-174. Sur la métamorphose entre le poisson et le dragon, voir en particulier les textes $n^{\circ} 3.31-39$ sélectionnés et traduits par Diény 1987, p. 77-81. Pour ce qui est de la monture des Immortels voir infra, nos citations du Shanhaijing et du Liexianzhuan. 
rencontre avec le mingshi 明師, le «maître éclairé » qui dispose des écrits et des renseignements authentiques et permet ainsi d'accéder à la tradition secrète 5 . Après avoir éprouvé la qualité du disciple et son aptitude à devenir un Immortel, le maître lui transmet les recettes et les écrits. Cependant, $\mathrm{Ge}$ Hong, qui cite le Huangdi jiuding shendan jing 黃帝九鼎神丹經, Livre des élixirs des neuf tripodes de l'Empereur jaune, nous apprend qu'avant de recevoir l'enseignement, le disciple prête serment devants les divinités : il doit, pour ce faire, lancer une figurine d'homme et une autre de poisson en or dans un cours d'eau coulant vers $l^{\prime}$ est $^{6}$. Selon le premier chapitre du Huangdi jiuding shendan jingjue 黃帝九鼎神丹經訣 qui serait le Huangdi jiuding shendan jing que connaissait Ge Hong, les frais de ces statuettes en or sont à la charge de celui qui reçoit l'enseignement. La première doit peser neuf liang 兩, la seconde trois. Après ou avant le jet des figurines, il faut aussi effectuer les pratiques de purification, puis établir, dans un endroit complètement inhabité près d'un courant d'eau, un siège (ou un autel) destiné à Xuannü 玄女 (une divinité de qui l'Empereur jaune a obtenu la recette), et annoncer au Ciel en brûlant l'encens : « Je voudrais transmettre la Voie de longue vie à ... (nom du disciple) ». Il faut ensuite placer le Livre des élixirs sur une tablette et celle-ci sur le siège. Quand on est prêt à transmettre la Voie, on se dirige vers le nord et on se prosterne pendant un moment. Si le ciel demeure clair et qu'il n'y a pas de vent, on peut transmettre la recette. En signe de serment, le maître et le disciple boivent ensemble le sang d'un coq blanc ${ }^{7}$. Selon le Jinyejing 金液經, le Livre de la liqueur d'or, cité aussi dans le Baopuzi neipian, il faut également, lors de la réception des "instructions orales", effectuer un rite analogue à celui que nous venons de décrire : offrir une figurine anthropomorphe en or de huit liang en la lançant dans un cours d'eau coulant vers l'est et boire du sang en signe d'engagement. C'est seulement après ce rituel que l'instruction peut être prononcée. Le Livre de la liqueur d'or précise que l'élixir ne pourra aucunement se former si on manque de procéder à cette cérémonie ou si on compose l'élixir à partir d'une recette "volée ", qu'il arrivera malheur au maître et au disciple si le Livre est transmis à la légère, et que les divinités célestes surveillent de près les hommes, à leur insu ${ }^{8}$.

Concernant la recette de Taiqing 太清, Ge Hong ne parle que des précautions à prendre contre une divulgation indue ${ }^{9}$. En revanche, nous trouvons quelques détails sur la transmission de la recette dans le Taiqingjing tianshi koujue 太清經天師口訣, qui date des Six Dynasties ${ }^{10}$. L'auteur de ces Instructions signale que ces dernières se fondent sur le

\footnotetext{
${ }^{5}$ Baopuzi neipian, 4/72.2, 12/226.4 et ailleurs.

${ }^{6}$ On ne connaît pas avec précision le moment du jet des figurines, qui se situe peut-être entre la pratique de purification et l'annonce au Ciel, ou entre la permission du Ciel et l'enseignement du maître. Cf. Baopuzi neipian, 4/74.5. Dans le Baopuzi shenxian jinzhuo jing, zhong/2b.1, l'expression de la figurine d'homme est omise.

${ }^{7}$ Huangdi jiuding shendan jingjue, 1/1b.2-8.

${ }^{8}$ Baopuzi neipian, juan 4, 3.13-15.

${ }^{9}$ Baopuzi neipian, juan 4, 76.11 .

${ }^{10}$ Pregadio 1991, p. 571-574.
} 
Taiqing shendan jing 太清神丹經 ${ }^{11}$, 1'un des premiers écrits alchimiques chinois, aujourd'hui perdu, mais qui est cité dans le Baopuzi neipian. Il souligne d'abord que la transmission doit s'effectuer oralement «pas à pas ", c'est-à-dire en stipulant jusqu'aux moindres détails (yi yi kou shou 一一口 授), et non pas «d'un seul coup» (et simplement) par un document (bu dei $d u n y i$ wen ye 不得頓以文也). Suivant un certain kemengshu 科盟書, «Ecrit du code et du serment», on dépense, en signe de foi, huit liang 兩 d'or, quarante chi 尺 de tissu jaune de fibre végétale, huit liang d'argent blanc et quarante chi de soie blanche. En signe du serment de ne pas divulguer la recette, on se frotte la bouche avec du rouge (probablement du sang ou du cinabre) ${ }^{12}$.

Le jet du poisson apparaît également dans certains textes de l'école Shangqing 上清. Selon le Dongzhen shangqing kaitian santu qixing yidu jing 洞真上清開天三圖七星移度經, qui précède le Wushang biyao 無上秘 要 du $6^{\mathrm{e}}$ siècle $^{13}$, on prépare un poisson d'or (jinyu 金魚) et un dragon de jade (yulong 玉龍) (5b.5) pour le rite de transmission des écrits jing. Le Zhen'gao 真誥 (8.13b.5) affirme que le don d'un dragon d'or et d'un poisson de jade (jinlong yuyu 金龍玉魚) est indispensable pour recevoir l'enseignement de la Voie. Cependant, ces textes ne nous renseignent pas sur les modalités d'usage de ces objets, par exemple où et comment les utiliser.

Eau

Certaines interrogations se posent autour du geste rituel du jet du poisson : sur ce que représente le poisson dans le symbolisme chinois ou taoïste et sur le rapport entre le poisson et les Immortels. Nous verrons que le poisson symbolisait, entre autres, le moyen du voyage ou de la transmission : il remplit le rôle d'attelage ou de messager entre ce monde et le monde des Immortels divins. Commençons par nous occuper de la relation qui existe entre le poisson et le monde des Immortels. Il nous semble qu'elle se manifeste, sur le plan symbolique, par l'intermédiaire de l'eau.

Dans l'Antiquité, les Chinois croyaient en des divinités des fleuves ou des rivières. La plus connue est Hebo 河伯, le Comte du fleuve Jaune. On lui offrait des sacrifices animaux ou humains, on jurait sur lui et les serments s'accompagnaient du jet d'un gage, l'anneau de jade par exemple ${ }^{14}$. Dans les Jiuge 九歌, les Neuf chants du Chuci 楚辭, Hebo est décrit monté sur un shuiche 水車, «char d'eau », tiré par deux dragons. En sa compagnie, le poète fait l'ascension du mont Kunlun et sur le chemin du retour, après s'être

\footnotetext{
11 Taiqingjing tianshi koujue, $1 \mathrm{~b} .2$.

12 Taiqingjing tianshi koujue, 1a.4-7. Le sang d'un animal blanc, la soie blanche et l'argent (donc blanc aussi) sont également employés en signe de serment dans la transmission de l' « instruction orale de l'Un véritable », ou de la «pratique de Garder l'Un». Voir Baopuzi neipian, juan 18, 324.15-325.1.

13 Voir Robinet 1984, p. 165 ; Lagerwey 1981, p. 1.

14 Voir Granet 1926, p. 466-482 ; Shanhaijing, p. 316-318.
} 
séparé de la divinité, il est escorté par des poissons ${ }^{15}$. Le Liexianzhuan 列仙 傳 présente des légendes qui relient les Immortels avec les poissons transportant les signes, les talismans ou les recettes ${ }^{16}$. Ge Hong nous apprend que, dans les montagnes, celui qui s'appelle Hebo est en fait l'esprit d'un poisson ${ }^{17}$.

Notre sujet doit aussi avoir un rapport, nous semble-t-il, avec le symbolisme du monde aquatique lié au monde invisible ou à la révélation de la norme ou du modèle par les réalités cosmiques. L'eau sépare, mais elle met aussi en liaison deux contrées. Selon la représentation chinoise, l'eau marque toujours la frontière entre le monde séculier et celui des Immortels. Cela va de soi si ce dernier se situe au milieu de la mer Orientale, au niveau de l'île de Penglai par exemple. Si le monde des Immortels se trouve au pic sacré de l'Ouest, au Mont Kunlun, l'eau qui l'entoure parvient jusqu'au monde d'ici-bas par des rivières ou des eaux souterraines ${ }^{18}$. Autrement dit, c'est à travers l'eau que l'accès à l'au-delà est possible ${ }^{19}$. On peut aussi évoquer la révélation du Hetu 河圖, le Tableau du Fleuve, ou le Luoshu 洛 書, l'Ecrit de la rivière Luo. Rappelons que les chevaux étaient sacrifiés au Fleuve et que le Hetu sortit du Fleuve sur le dos d'un cheval-dragon ${ }^{20}$. Dans l'école des Maîtres Célestes, on croyait en Sanguan 三官 : le Ciel, la Terre et l'Eau divinisés, auxquels on s'adressait au cours de l'une des cérémonies de purification. Il existe aussi un autre symbolisme lié à la cosmologie chinoise dans lequel l'eau, appelée neijing 内景, « luminescence interne », représente le principe Yin et la transformation potentielle ${ }^{21}$.

Poisson

Le poisson, de même que le dragon, la tortue ou le crapaud, représente le monde aquatique ${ }^{22}$. Et il fait partie du symbolisme de l'intermédiaire entre ce monde et le monde des Immortels divins. En particulier, il apparaît dans

15 Chuci, 6:1.23-26. Waley 1955, p. 47.

16 Liexianzhuan, textes $\mathrm{n}^{\circ}$ XI, XII, LXVII, LXVIII.

17 Baopuzi neipian, juan 17, 304.8 .

18 Le mont Kunlun constitue l'un des mondes des Immortels et correspond à un axis mundi chinois. C'est une ville d'en bas pour les dieux, les $d i$ 帝 selon le Shanhaijing, ch. 11, 294.6. Il est entouré d'une « eau molle» (ruoshui zhiyuan 弱水之淵) : Shanhaijing, ch. 16, 407.13. Il y a neuf puits : Shanhaijing, ch. 11, 294.7 ; Huainanzi, juan 4, 133.12 ; Lüshi chunqiu, «Benwei », 741.8-9. Quatre rivières, issues du mont Kunlun, sont les sources divines des $d i$, qui se mélangent avec toutes sortes de remèdes et humectent les milles êtres : Huainanzi, juan 4, p. 134-135. Les neuf collines où se trouve l'arbre jianmu 建木, qui est l'arbre cosmique et le centre du monde, sont également entourées d'eau : Shanhaijing, ch. 18, p. 448. Voir aussi Allan 2001, p. 29.

19 D'après Allan 1991, p. 16, les gens de l'époque des Shang auraient placé sur les autels ancestraux ou immergé dans les cours d'eau les promesses rituelles ou serments inscrits sur les fiches de bambou.

20 Granet 1926, p. 476-478.

${ }^{21}$ Huainanzi, juan 3, 80.14.

22 Pour une représentation Han du monde aquatique, voir infra (note 44), celle du Wuliangci, présentée par Chavannes. 
de nombreuses images Han décrivant des aspects du monde des Immortels. Sur ces images, il fait corps avec les éléments caractéristiques du monde des Immortels, par exemple avec les Immortels eux-mêmes ${ }^{23}$.

Commençons par citer une légende racontée dans le Liexianzhuan :

Qin Gao était un homme du pays de Zhao. Parce qu'il jouait bien du tambour et du luth, il fut intendant du roi Kang de Song; il pratiquait les recettes de Juanzi et de Pengzu. Il voyagea dans la région située entre le Jizhou et la commanderie de Tang durant plus de deux cents années. Plus tard, il se retira et entra dans les eaux de la rivière Dang où il prit de petits dragons (qu long $z i$ 取龍子). Il fixa un rendez-vous à ses disciples et leur recommanda de se purifier, de jeûner (jiezhai 潔齋) et de l'attendre au bord de la rivière où ils devaient élever un sanctuaire (sheci 設祠). En effet, [le jour venu] il arriva monté sur une carpe rouge (cheng chi li 乘赤鯉) et, sortant [des eaux], alla s'asseoir au milieu du sanctuaire. Le lendemain à l'aube, dix mille personnes vinrent le contempler. Il resta plus d'un mois, puis il repartit sur les eaux de la rivière ${ }^{24}$.

Qin Gao est tout d'abord un homme qui pratique les méthodes visant à obtenir la longue vie et qui finit par l'obtenir ${ }^{25}$. Il entre ensuite dans les eaux et attrape (un ou) des dragons ${ }^{26}$. Ensuite, et c'est cela qui nous intéresse le plus, il ressort de l'eau, Immortel monté sur une carpe. Il est à noter que dans ce texte antérieur aux Chapitres Intérieurs, la légende indique un prototype (ou une naissance) du culte des Immortels, prototype que nous remarquons aussi dans le rite de transmission décrit par Ge Hong. Dans la même hagiographie, nous retrouvons des motifs analogues, notamment : un pêcheur qui s'élève dans le ciel sur le dos d'un poisson ailé27 ; un forgeron, maître ou éleveur de dragons, qui part sur le dos d'un dragon ${ }^{28}$.

La représentation pisciforme du moyen d'atteindre le monde des Immortels nous paraît significative, car c'est le sens que nous sommes tenté de donner à la figurine du poisson que l'on jette dans les eaux. D'ailleurs, cela est suggéré par certaines représentations des tombes des Han. Dans ces

${ }^{23}$ Li Falin 1982, tableau $n^{\circ} 18$; Jining diqu wenwu zu et Jiaxiangxian wenguansuo 1982, p. 65. Voir aussi la figure 1 tirée de Liaocheng diqu bowuguan 1989, p. 53 : il s'agit du dessin gravé sur la face est du pilier central de la porte de la salle postérieure de la tombe $n^{\circ} 1$ de Balimiao 八里廟 dans le district de Yanggu 陽谷 (Shandong), découverte en 1983. Autre exemple : trois poissons sont placés au-dessus des oiseaux et des Immortels dans l'une des scènes des pierres gravées découvertes au sud de la ville de Jining 濟寧 (Shandong), présumées dater des Han Orientaux : Jiningxian wenhuaguan et Xia 1983, p. 25, fig. $n^{\circ} 9$. L'association symbolique de l'oiseau et du poisson y est fréquente. Elle représente la venue de la richesse et de la prospérité, les caractères $y u$ 魚 pour le poisson et le $y u$ 裕 pour l'abondance étant homophones.

24 Kaltenmark 1953 tr., p. 105. Pour une autre traduction voir Diény 1987, p. 78.

25 Qin Gao est aussi mentionné dans le Baopuzi neipian, juan 3, 49.6, voir infra.

26 Pour l'Immortel sous l'apparence d'un dragon, voir Diény 1987, textes n 2.79 et 3.41 .

27 Liexianzhuan, texte $\mathrm{n}^{\circ} \mathrm{LV}$.

${ }^{28}$ Liexianzhuan, textes ${ }^{\circ}$ III, V, XLV, LX. Par ailleurs, selon Needham et al. 1976, p. 8, la métaphore de "tuer le dragon », associée à la formation de la chaux ou du sulfure d'un métal, est commune à l'alchimie du monde entier. Le poisson est aussi associé au travail du fondeur : Stein 1947, p. 242, 249. 
images, aussi peu nombreuses soient-elles, le poisson a une fonction : il est l'un des véhicules entre ce bas monde et le monde des Immortels divins, comme nous le verrons un peu plus loin.

Auparavant, il s'avère nécessaire de cerner le contexte dans lequel se situent les images que nous allons aborder. Ce sont des images qui décorent les tombes des Han. Ces dernières sont particulièrement riches en images gravées sur les pierres murales intérieures des tombes. Elles présentent, en divers styles, la notion du monde d'outre-tombe, voire du monde des Immortels. Grâce à l'art funéraire, nous apprenons que la tombe, dernière demeure de l'homme, était conçue comme un endroit où les défunts continuaient, ou arrivaient enfin, à jouir du bonheur. A l'époque des Royaumes Combattants, les Chinois construisaient les tombes de façon à ce que les «âmes », qui continuent à exister après la mort, puissent y vivre comme avant la mort. Autrement dit, ils leur offraient une dernière demeure bienheureuse. Il est probable qu'à cette époque, ils considéraient que l'immortalité ne pouvait être acquise que pendant la vie, avant la mort. A partir des Han, l'idée de la vie éternelle après la mort apparaît progressivement dans l'art funéraire. Ainsi, tout en étant des «maisons heureuses ", les tombes de l'époque des Han sont ornées d'images qui évoquent un lieu de passage et représentent l'arrivée du défunt dans le monde des Immortels 29 .

La tombe que nous avons choisie comme premier exemple illustre bien cet aspect. Elle a été découverte au nord du village de Chengqian 城前, à 1,5 kilomètres à l'ouest du district de Cangshan 蒼山, dans la province du Shandong, et elle date très probablement de 151 après J.-C. ${ }^{30}$. Sa structure comprend deux parties: une antichambre et une chambre principale par rapport à l'entrée de la tombe. La décoration murale de l'antichambre, là où transitait le cercueil, représente le voyage funéraire, intercalé dans le temps entre la vie en ce monde et celle dans l'au-delà ${ }^{31}$. Elle est constituée de scènes de processions funéraires. Cette salle correspondrait donc à la phase de l'enterrement et du début de la vie souterraine ${ }^{32}$. La chambre principale, où était déposé le cercueil, correspond à la phase suivante ${ }^{33}$. Elle est encadrée par des scènes du monde céleste et mythique et du voyage dans (ou vers) ce monde céleste, scènes qui matérialisent la vie du défunt dans l'audelà.

Cette tombe recèle une inscription, gravée sur l'un des piliers d'entrée de la petite pièce annexe, sur le côté ouest de l'antichambre. Elle explique le plan architectural et pictural de la tombe ${ }^{34}$. L'auteur — probablement à la

${ }^{29}$ Wu Hung 1994, 82-88.

${ }^{30}$ Voir le rapport de fouilles : Shandongsheng bowuguan et Cangshanxian wenhua guan 1975.

${ }^{31}$ Nous adoptons ici la lecture de Wu Hung (1994, p. 102) qui n'est pas la seule qui a été proposée.

32 Wu Hung 1994, p. 100-101. Voir la figure du Shandongsheng bowuguan et Cangshanxian wenhua guan 1975, p. 129.

33 Pour la disposition, voir le rapport de fouilles : Shandongsheng bowuguan et Cangshanxian wenhuaguan 1975, p. 125.

34 Wu Hung 1994, p. 92. Voir la figure du Shandongsheng bowuguan et Cangshanxian wenhuaguan 1975, p. 126. 
fois du texte et de la décoration de la tombe ${ }^{35}-\mathrm{y}$ décrit principalement le « voyage » du défunt depuis sa mort. C'est ce texte qui contient le mot-clé sur lequel nous nous appuyons pour proposer notre interprétation de la figurine du poisson en or. C'est l'expression : jialiyu 駕鯉魚, « conduire les carpes attelées ». La scène de ces carpes n'est pas figurée dans la tombe, mais, selon le texte, elle devrait se trouver au plafond de la chambre principale et faire partie de l'ensemble de la représentation du monde de l'au-delà ${ }^{36}$. Les images destinées à encadrer cette salle étaient toutes mythiques, transformant pour ainsi dire la salle en un microcosme ${ }^{37}$. Voici une partie du texte qui explique ces images :

$\mathrm{Au}$ vingt-quatrième jour du huitième mois de la première année de l'ère Yuanjia (151 après J.-C.), nous achevons la construction de cette pièce de la tombe pour vous envoyer, membre honorable de la famille, en voyage. $\mathrm{Si}$ votre âme possède la connaissance, veuillez avoir pitié de vos descendants, qu'ils prospèrent dans leurs moyens d'existence et obtiennent la longévité.

[Permettez-nous de vous] montrer et expliquer les images de la tombe.

Sur le mur postérieur (dans la chambre principale) : le Faisan Rouge rencontre un Immortel qui flâne. Les Phénix suivent le Tigre Blanc qui se promène au milieu.

Sur la colonne centrale (en façade de la chambre principale) : un couple de dragons entrelacés garde le cœur de la tombe et détourne le mal.

Sur le plafond de la chambre [principale] : une voiture wuzi 五子 est suivie de jeunes servantes qui conduisent les carpes; au-devant court le char du Tigre Blanc et du Dragon Bleu; le Duc Tonnerre se trouvant sur les roues soulève l'arrière ; ses assistants qui poussent le char sont des renards et des canards mandarins ${ }^{38}$.

Dans ces images, la carpe apparaît, parmi les êtres célestes (ou cosmiques) et prodigieux, comme un véhicule, un moyen de voyage.

Nous pouvons ajouter à cette description quatre images provenant de tombes proches, dans le temps et dans l'espace, de celle de Cangshan, qui recoupent ce que nous venons de dire.

Une gravure sur pierre des Han, découverte près d'une tombe de la même période, présente la scène suivante ${ }^{39}$ : trois poissons tirent un char conduit par un Immortel (ou un génie) reconnaissable à ses ailes et à sa coiffure pointue. Un personnage, monté à l'arrière de ce char, porte sur la tête une sorte de coiffe en forme de poisson. À côté, il y a trois dragons tirant un

\footnotetext{
35 Wu Hung 1994, p. 93 et la note 16.

36 Selon Wu Hung (1994, p. 98), il est possible que la tombe ait été achevée à la hâte avant que la partie du plafond soit complètement décorée et que le texte servit à documenter le plan originel de la tombe.

37 Wu Hung 1994, p. 100.

38 Sur le déchiffrage et la ponctuation du texte, les études et les opinions divergent. Nous avons suivi la traduction de Wu Hung 1994, p. 93, qui a pris plusieurs études en considération.

39 Voir la figure 2, tirée du Jiangsusheng wenwu guanli weiyuanhui 1959, fig. $n^{\circ} 52$.
} 
autre char ${ }^{40}$. C'est une scène de défilé précédé par un cracheur de feu. Manifestement, ce défilé se dirige vers un personnage qui l'accueille. L'ensemble de cette image nous semble vouloir évoquer le voyage du défunt vers le monde des Immortels. Cette pierre se situe à Honglou 洪樓, dans le district Tongshan 銅山 de la préfecture Xuzhou 徐州 de la province du Jiangsu, limitrophe à la fois de la province du Shandong et de celle de l'Anhui ${ }^{41}$.

Nous trouvons un autre groupe de trois poissons attelés à un char, gravé sur une dalle du mur est de l'antichambre d'une tombe Han, découverte en 1956 au village Luanzhen 戀鎮, à cinquante kilomètres environ au sud-ouest du district de Feicheng 肥城 dans la province du Shandong ${ }^{42}$. Un cheval et un cerf tirent chacun un char, le premier devant celui des poissons, l'autre, derrière. Dans cette scène complexe, représentant à la fois des aspects séculiers et cosmiques, nous trouvons également, à gauche et à droite du bâtiment central, Fuxi 伏羲 et Nüwa 女媧, divinités cosmiques mâle et femelle souvent accompagnées par le soleil et la lune, symbolisant l'opposition et l'harmonie du Yin et du Yang, tenant ici en main le compas et l'équerre.

Signalons aussi une image appartenant à un groupe de tombes, connu depuis l'époque des Song sous le nom de «chambres d'offrandes de la famille $\mathrm{Wu} »$. Ce sanctuaire se trouve à $20 \mathrm{~km}$ environ au sud du district Jiaxiang 嘉祥, dans l'ouest de la province du Shandong. Les textes épigraphiques nous indiquent que ces tombes de la famille $\mathrm{Wu}$ datent du milieu du deuxième siècle après J.-C. ${ }^{43}$. Nous empruntons à Chavannes la description de l'image qui nous intéresse : "Il semble qu'on soit dans le royaume des eaux. Sur un char tiré par deux poissons est assis un grand personnage tenant un insigne " fou » : derrière lui est son cocher; en arrière du char, un homme debout, et, en avant du char, un homme agenouillé, présentent tous deux la tablette «hou». Autour de ce groupe principal s'agite tout un cortège étrange : (...) tout un peuple de poissons. (...) Je propose de reconnaître dans le personnage principal qui est assis dans le char la divinité la plus populaire parmi les divinités aquatiques, le fameux comte du Fleuve qui préside au Houang ho $(\ldots) »^{44}$.

La dernière image que nous voulons présenter diffère des images précédentes car le poisson n'est pas attelé à un véhicule : une divinité (ou un Immortel) ailée se tient debout sur son dos. Un autre être, dessiné à côté dans la même scène, chevauche une grue, animal emblématique associé aux Immortels et à la longue vie. La tombe qui recèle cette image date d'une époque tardive des Han Postérieurs et se trouve au village Wangcun 王村, à

40 Selon le Shanhaijing, Bing Yi 冰夷 conduit deux dragons. Les commentaires nous enseignent que Bing Yi 冰夷 ou Feng Yi 馮夷 serait un Immortel (ou une divinité) du fleuve, c'est-à-dire un Hebo. Voir Shanhaijing, ch. 12, 316.10-318.13.

41 Jiangsusheng wenwu guanli weiyuanhui 1959, p. 10.

42 Wang Sili 1958, p. 35. Voir la figure 3 tirée du même article.

43 Concernant les dates, voir Wu Hung 1989, p. 24-30. A propos de l'emplacement de l'image en question, voir Wu Hung 1989, p. 15-24.

44 Chavannes 1913, p. 207-208. Voir la figure 4 tirée de Chavannes 1909, n 130. 
$15 \mathrm{~km}$ au nord du district de Xia 夏縣, dans la province du Shanxi ${ }^{45}$. En dehors de l'iconographie, on trouve également le couple poisson/oiseau dans la littérature. On lit dans un passage du Baopuzi neipian : "Qui a trouvé la Voie peut élever son corps jusqu'aux cieux, s'immerger dans la mer et les rivières. Xiao Shi, qui s'éleva dans les airs en compagnie d'un phénix, et Qin Gao, qui plongea dans les profondeurs sur le dos d'une carpe rouge, nous en apportent la preuve. ... $\gg^{46}$.

Ainsi, dans l'art funéraire Han, le poisson est associé aux Immortels et fait vraisemblablement partie du symbolisme du voyage céleste.

En dehors de la tombe, le poisson, souvent la carpe, symbolise aussi le messager miraculeux. Dans les histoires légendaires recueillies dans les textes datant des premiers siècles de notre ère, le poisson, envoyé souvent par une divinité, bondit hors de l'eau et se retrouve face à un personnage en chemin ou déjà arrivé (chez lui) ; quand il ouvre le ventre du poisson, il trouve l'objet qui lui est destiné ${ }^{47}$. Vraisemblablement, cette croyance dans le rôle d'intermédiaire joué par le poisson a un rapport étymologique avec une expression chinoise employée jusqu'à nos jours, qui désigne le courrier : $l i s h u$ 鯉書 (mot à mot, carpe - écrit) ou lisu 鯉素 $\square$ (carpe - étoffe de soie écrue, sur laquelle on écrivait) ou encore liyu chisu 鯉魚尺素 $\square$ (carpe poisson - pied (mesure) - étoffe de soie, ou carpe (liyu) - lettre $(\text { chisu) })^{48}$.

Par ailleurs, de nombreux insignes en deux parties étaient en forme de poisson $^{49}$. Selon Shavkunov (1989), une paire de plaques de bronze en forme de poisson avec inscription, découverte dans la région de Nikolayevsk en Russie, serait une œuvre des Tang du $8^{\mathrm{e}}$ au $9^{\mathrm{e}}$ siècle ou du royaume de Bohai 渤海 (698-926) de la même période. Il présume qu'une partie était possédée par un ministre militaire résidant dans la région limitrophe et l'autre, par l'Etat.

Nous voyons donc que le poisson constitue couramment chez les Chinois le symbole du moyen de transmission par excellence, circulant de l'un à l'autre, d'un monde à un autre.

\section{Jet du dragon}

\footnotetext{
45 Voir la figure 5 : Shanxisheng kaogu yanjiusuo et al. 1994, p. 36.

46 Baopuzi neipian, 3/49.5-6. Traduction de Che 1999, p. 85. Pour une interprétation de ce passage qui porte sur la dialectique de "l'ascension céleste et de la descente dans la Terre », voir Robinet 1996, p. 175-176. Selon un contrat d'achat de terre, inscrit sur une tablette et daté de 962 après J.-C., la grue monte vers le Ciel bleu et la carpe s'enfonce dans les profondeurs des Sources pour informer les divinités de ce contrat: Niida 1937, p. 111 ; Gernet 1957, p. 337, n.1.

47 Voir Miyakawa 1979, p. 86-88. Voir aussi le Liexianzhuan, textes ${ }^{\circ}$ XI, XII, LXVII, LXVIII.

48 De même, voir le Gu yuefu, «Yinma Changcheng kuxing 飲馬長城窟行 (Sur le chemin, en faisant boire le cheval [à l'eau de] la source-grotte [au-dessous] de la Grande Muraille) », p. 491 : Un hôte venant de loin laisse une paire de carpes. Le maître de la maison les fait cuire par un jeune serviteur. [Quand on les ouvre,] on trouve à l'intérieur une missive (chisushu 尺素書).

${ }^{49}$ Voir des Rotours 1952.
} 
Etant donné que le jet du dragon s'insère, comme le jet du poisson, dans des contextes rituels et qu'il s'effectue explicitement en vue de faire parvenir l'intention de l'homme auprès des divinités célestes, une mise en parallèle avec le jet du poisson nous conforte dans l'interprétation du rôle symbolique du poisson. En relevant le contexte et la circonstance rituels de l'acte afin de le rapprocher du jet du poisson, nous constatons que :

1) au début, le jet du dragon était associé au rite de transmission du livre secret de maître à disciple, et progressivement, il a été associé à des rites à but divers, mais toujours pour son rôle d'envoyer un message vers l'au-delà ;

2) de manière générale un serment est associé à cet acte rituel ;

3) parfois les éléments de sanguan shoushu continuent à apparaître, parfois il n'y a que le jet du dragon dans l'eau.

Selon les études précédentes, comme nous l'avons déjà mentionné tout au début de l'article, le geste rituel résulte d'une évolution des rites sacrificiels exécutés par le souverain dans la Chine antique et, plus directement, de la méthode des sanguan shoushu, de communiquer les «documents adressés aux Trois Officiers (Ciel, Terre, Eau)»du mouvement des Cinq boisseaux de riz des Han Postérieurs ${ }^{50}$. Au cours de ces pratiques, les Chinois jetaient des objets dans les eaux ou les enterraient en destination des divinités. Cependant, on ignore les formes spécifiques sous lesquelles se présentaient ces objets.

Les premiers textes dans lesquels nous pouvons constater le rite du jet du dragon sont ceux de la tradition Lingbao qui dateraient d'avant le $5^{\mathrm{e}}$ siècle après J.-C. ${ }^{51}$. D'après le Taishang dongxuan lingbao chishu yujue miaojing 太上洞玄靈寶赤書玉訣妙經, pour communiquer avec la bureaucratie divine, il convient de jeter un dragon en or chargé de «tablettes », jian 簡, dans les lacs des trois fleuves, sanhe zhiyuan 三河之淵; d'en enterrer un sur une « montagne merveilleuse », lingshan 靈山, à laquelle appartient le destin originel, benming 本命, de l'adepte; et d'en enterrer un troisième pour l'envoyer au palais du milieu, zhonggong 中宮 52 .

Cependant, par rapport au rite de sanguan shoushu, consistant à envoyer un message aux divinités en vue d'une guérison, d'une purification des adeptes malades ou d'une confession pour obtenir un acquittement, le jet du dragon, lié aux rites Lingbao, est différent d'une part pour ce qu'il représente, et d'autre part du point de vue du contexte rituel dans lequel il s'insère. En premier lieu, le dragon incarne l'esprit qui est reconduit lors de la clôture des rites comme le Jinluzhai 金銾齋 ou le Huangluzhai 黃錄齋, organisés à la demande de «clients » laïcs et pendant lesquels les officiants taoïstes prient pour le bonheur des vivants ou des défunts ${ }^{53}$. En second lieu, et c'est le cas qui nous intéresse, le jet du dragon exprimant le serment et la sincérité de l'adepte ou de l'officiant, est associé à la transmission des écrits sacrés et des

\footnotetext{
50 Voir supra.

51 Ôfuchi Ninji 1974, 45 s. ; Bokenkamp 1983, appendix.

52 Taishang dongxuan lingbao chishu yujue miaojing, shang, 5a.3-7b.8.

53 Yamada Toshiaki 1999, p. 187-188.
} 
registres rituels ${ }^{54}$. Selon une formule citée dans un texte de Lu Xiujing 陸修 靜(406-477), le Taishang dongxuan lingbao shoudu yi 太上洞玄靈寶授度 儀, le jet du dragon, tout en gardant une triple destination, fait partie du rite de transmission des textes sacrés, des registres rituels et des recettes, relatifs à la quête de l'immortalité 55 .

Dans les textes postérieurs, il est inséré dans des rites taoïstes plus variés tout en conservant les expressions récurrentes qui accompagnent le geste, par exemple : yuanshen yuanxian 願神願仙, « souhaiter devenir divin, devenir immortel »; jinlong yichuan 金龍驛傳, « que les courriers des dragons d'or transmettent cela », qui se trouvaient déjà dans le Taishang dongxuan lingbao chishu yujue miaojing.

A partir de la dynastie des Tang, favorisée par les empereurs et leur famille, cette activité rituelle s'établit comme un rite protocolaire de l'Etat. Elle s'associe au rite sacrificiel rendu aux monts et aux eaux, lequel était jadis celui des Fils du Ciel. Zhou Xibo a examiné le sujet afin de cerner dans quelles circonstances a été rédigé le manuscrit de Dunhuang P.2354. D'après ce manuscrit, il pense que l'activité, devenue un protocole promulgué par l'Etat, était répandue partout dans la Chine des Tang. Sous les Ming, le jet du dragon se pratiquait toujours ${ }^{56}$.

A partir des renseignements puisés dans les études précédentes, on peut établir les quatre points distinctifs qui permettent de rapprocher le jet du dragon du jet du poisson :

1) le jet du dragon était souvent accompagné de l'envoi d'un message écrit ;

2) la figurine-dragon était considérée comme un messager ou un guide $^{57}$ transportant les mots écrits au destinataire ${ }^{58}$;

3) le lieu où l'on pratiquait cette cérémonie était censé communiquer avec le monde des Immortels ;

4) l'acte s'effectuait au moment de la transmission des écrits ou des recettes.

Prenons deux exemples. Dans son message, jeté avec des dragons en métal doré en 738, Xuanzong (r. 712-756) se désigne par son nom de famille et son prénom ${ }^{59}$. Il indique aussi sa date de naissance ${ }^{60}$. Les dragons sont considérés comme des coursiers qui transmettent son écrit impérial. La

54 Liu Zhaorui 2001, p. 485 ; Zhou Xibo 1999, p. 92 ; Schipper 1991, p. 227; Yamada Toshiaki 1999, p. 283.

55 Taishang dongxuan lingbao shoudu yi, 3b.6-7. Voir aussi le Taishang dongxuan lingbao zongjian wen, 2a.3-4.

56 Wang Yucheng 1994 ; Zhou Xibo 1999, p. 104-106.

57 Pour le dragon-guide, voir Kaltenmark 1948, p. 60-61.

58 Sur des thèmes analogues, voir Diény 1987, section VI-7, «Un cheval céleste », p. 199203.

59 Chavannes 1919, p. 55-58 ; Liu Zhaorui 2001, p. 478-480. Dans son texte du jet du dragon jeté en 845, Wuzong (r. 840-846) se désigne par une appellation taoïste : Liu Zhaorui 2001, p. 480.

60 Chavannes (1919, p. 57) présume que c'est afin de permettre aux magistrats souterrains qui président à la durée des destinées humaines de retrouver son nom dans le registre des naissances et des morts. 
cérémonie a été accomplie dans la " grotte des Immortels » du pic du Zigai 紫蓋 ou Dais Violet, du Mont Heng (Hengshan 衡山) ${ }^{61}$. Dans la pensée chinoise, la grotte est ouverte non seulement au cœur de la terre mais aussi au ciel et au monde des Immortels ${ }^{62}$. Soulignons qu'une « méthode » est liée au rite. L'empereur allait la recevoir ou venait de l'obtenir, puisque le texte dit qu'il «souhaitait en profiter». Comme nous l'avons vu plus haut, cela ressemble au devoir du disciple alchimiste d'effectuer la cérémonie avant la transmission des recettes dont parle Ge Hong. En l'an 928, Qian Liu 錢鏐, roi de $\mathrm{Wu}$ et de Yue, accomplit la cérémonie du jet des dragons dans le lac Tai (Taihu 太湖) ${ }^{63}$. La prière, que nous ne citons pas, est analogue au message de Xuanzong. Elle demande le bonheur pour lui et pour la dynastie, ainsi que la longévité. Le dragon fait fonction d'escorte, non pas sous forme de statuette, mais en encadrement dessiné autour du texte. Selon un récit, on a trouvé au même endroit plusieurs statuettes de dragon ${ }^{64}$. Tout en s'adressant aux divinités célestes, il jette dans les eaux son message qui va arriver dans leur résidence aquatique.

En définitive, au regard de leur but aussi bien que de point de vue morphologique, se ressemblent l'acte du disciple alchimiste dont parle Ge Hong et la cérémonie du jet du dragon ainsi que le rôle du " poisson », jeté avec «l'homme », et le rôle du "dragon », jeté avec "un message ». Par conséquent, l'acte du disciple alchimiste signifierait que «l'homme » émis de la main de l'initié sera emmené jusqu'au monde des Immortels divins par «le poisson ».

Dans le taoïsme, les textes secrets ou destinés à être révélés uniquement aux personnes jurées sont, à l'origine, conservés dans la demeure des divinités ou des Immortels divins. Revêtus d'une forme intelligible à l'homme, ils constituent un autre aspect des talismans, charmes, gages d'alliance ou mandats célestes. Un élixir alchimique a d'ailleurs pour titre shenfu 神符, «talisman divin ${ }^{65}$. La transmission de ces « écrits » précieux chargés de pouvoirs exige un engagement, un crédit ${ }^{66}$. Ainsi, l'offrande rendue met-elle l'homme en relation, par une alliance, avec les divinités. Les

61 A propos de cette grotte, voir Liu Zhaorui 2001, p. 479.

62 Stein 1943, p. 62-64. Voir aussi Chavannes 1919, p. 96-97: selon une inscription de l'année 739 provenant de la montagne Dafang 大房山, le rite du jet des dragons y fut pratiqué dans une grotte Kongshui 孔水洞. Plus tard, sous les Ming, en parlant de cette grotte, Liu Tong 劉侗, dans son Dijing jingge lue 帝京景格略, dit avoir « vu en sortir un ruisseau des fleurs de pêcher, sans doute pour signifier que le ruisseau provient du pays des Immortels. Remarquons également dans ce passage l'idée de transformation entre le dragon et le poisson.

63 Chavannes 1919, p. 59-67 ; Liu Zhaorui 2001, p. 481-482.

64 Chavannes 1919, p. 60-61.

65 Baopuzi neipian, juan 4, 75.1.

${ }^{66} \mathrm{Au}$ sujet de la relation entre les écrits prodigieux ou précieux et le terme lingbao 靈寶, « joyau spirituel», voir Kaltenmark 1961. A propos de la transmission des textes précieux dans le taoïsme, voir aussi Stein 1968. 
images de poisson et de dragon dans une matière précieuse comme l'or ou le jade étaient employées pour représenter le rôle de médiation.

En particulier, le poisson figure, dans les peintures et les usages littéraires que nous avons examinés, le véhicule transmetteur entre deux mondes séparés. Hebo était d'ailleurs considéré comme un psychopompe qui mène les chamans ou les morts au mont Kunlun, l'une des résidences sur la terre des divinités célestes.

Pour revenir au jet du poisson qu'accomplit le disciple initié dans la tradition taoïste, tel que les ouvrages $\mathrm{du} 4^{\mathrm{e}}$ au $6^{\mathrm{e}}$ siècle le décrivent, nous serions en conclusion tenté de l'interpréter comme une mise en scène visant à envoyer dans le monde des Immortels divins la nouvelle d'une initiation, d'une transmission et d'un engagement, ou peut-être la personne même de l'initié. C'est un signe de serment, disait l'alchimiste taoïste, à l'égard des Immortels divins qui sont à l'origine de la recette, et un avertissement symbolique ; un signe admettant que l'initié est d'ores et déjà affilié à la société des Immortels. Pour le signaler, si notre interprétation est juste, il envoie son image escortée par un poisson apte à voyager jusqu'au monde des Immortels. Ces derniers seront ainsi informés de son initiation.

\section{Bibliographie}

Allan, Sarah. 1991. The Shape of the Turtle : Myth, Art, and Cosmos in Early China. Albany : State University of New York Press.

Baopuzi neipian. Ge Hong (283-343). Baopuzi neipian jiaoshi 抱樸子内篇 校釋, édité par Wang Ming 王明, Zhonghua shuju, 1985.

Baopuzi shenxian jinzhuojing 抱樸子神仙金汋經. Probablement des Tang, traditionnellement attribué à Ge Hong. Daozang (CT 917).

Bokenkamp, S.R. 1983. « Sources of the Ling-pao scriptures ». Mélanges chinois et bouddhiques, vol. 21 : Tantric and taoist studies in honour of R.A.Stein, vol. 2 : 434-486.

Chavannes, E. 1909. Mission archéologique dans la Chine septentrionale, Planches, ${ }^{\text {ère }}$ partie, Paris, Ernest Leroux.

Chavannes, E. 1913. Mission archéologique dans la Chine septentrionale, Tome I, Première partie : La sculpture à l'époque des Han. Paris, Ernest Leroux.

Chavannes, E. 1919. « Le Jet des dragons », Mémoires concernant l'Asie Orientale, Tome III : 54-220.

Che, P. 1999. Ge Hong. La voie des Divins immortels. Gallimard.

Chuci 楚辭. Contenus de dates variées du $4^{\mathrm{e}}$ siècle A.C. tardif au $2^{\mathrm{e}}$ siècle P.C. A Concordance to the Chuci, the Chinese University of Hong Kong, Institute of Chinese Studies, the Commercial Press, 2000.

Diény, J.-P. 1987. Le symbolisme du dragon dans la Chine antique. Paris, Collège de France, Institut des Hautes Etudes Chinoises. 
Dongzhen shangqing kaitian santu qixing yidu jing 洞真上清開天三圖七星 移度經. $6^{\mathrm{e}}$ siècle au plus tard. Le texte à l'origine de celui-ci serait l'un des premiers textes de l'école Shangqing. Daozang (CT 1317).

Gernet, J. 1957. «La vente en Chine aux 9-10 ${ }^{\mathrm{e}}$ siècles d'après les contrats de Touen-houang ». T'oung pao, XLV, 4-5 (1957) : 295-391.

Gu yuefu 古樂府. Des Yuan. Zuo Keming 左克明. In coll. de Qinding Siku quanshu, édition de Wenyuange, 1983, vol. 1368.

Granet, M. 1926. Danses et légendes de la Chine ancienne. Paris, Presses Universitaires de France, 1959. Edition originelle : Paris, Librairie Félix Alcan, 1926.

Huainanzi 淮南子. Liu An 劉安 (179-122 A.C.) et autres. Ed. de Liu Wendian 劉文典, Huainan honglie jijie 淮南鴻烈集解, in coll. de Xinbian Zhuzi jicheng, Beijing, Zhonghua shuju, 1989.

Huangdi jiuding shendan jingjue 黃帝九鼎神丹經訣. Le texte de l'édition du Daozang de l'ère Zhengtong (1436-1450) composé de vingt chapitres daterait de la seconde moitié du $7^{\mathrm{e}}$ siècle (CT 885).

Jiangsusheng wenwu guanli weiyuanhui 江蘇省文物管理委員會. 1959. Jiangsu Xuzhou Han huaxiangshi 江蘇徐州漢畫像石. Zhongguo kexueyuan kaogu yanjiu suo, Beijing, Kexue chuban she.

Jining diqu wenwu zu 濟寧地區文物組 \& Jiaxiangxian wenguansuo 嘉祥縣 文管所. 1982. «Shandong Jiaxiang Songshan 1980 nian chutu de Han huaxiangshi 山東嘉祥松山 1980 年出土的漢畫像石 》. Wenwu 文物, 1982/2:60-70.

Jiningxian wenhuaguan 濟寧縣文化館 et Xia Zhongrun 夏忠潤. 1983. 《Shandong Jiningxian faxian yizu Han huaxiang shi 山東濟寧縣發現一 組漢畫像石 》. Wenwu, 1983/5:21-27.

Kaltenmark, M. 1948. « Le dompteur des flots ». Han-hiue, vol. 3, no. 1-2 : 1-113.

Kaltenmark, M. tr. 1953. Le Lie-sien tchouan (Biographies légendaires des Immortels taoïstes de l'antiquité). Paris, Institut des Hautes Etudes Chinoises, 1987. Réimpression de l'édition originelle : Pékin, Centre d'études sinologiques de Pékin de l'Université de Paris, 1953.

Kaltenmark, M. 1961. « Ling-pao 靈寶: note sur un terme du taoïsme religieux ». Mélanges publiés par l'IHEC, tome 2 (1961) : 559-588.

Lagerwey, J. 1981. Wu-shang pi-yao. Somme taoïste du $6^{e}$ siècle. Paris, Ecole Française d'Extrême-Orient.

Li Falin 李發林. 1982. Shandong Han huaxiangshi yanjiu 山東漢畫像石研 究, Shandong, Qilushushe.

Li Ling 李零. 2000. Zhongguo fangshu xukao 中國方術續考. Beijing, Dongfang chubanshe.

Liaocheng diqu bowuguan 仰城地區博物館. 1989. 《Shandong Yangguxian Balimiao Han huaxiangshi mu 山東陽谷縣八里廟漢畫像石墓》. Wenwu, 1989/8 : 48-56.

Liexianzhuan 列仙傳. Des époques diverses selon le texte. Attribué à Liu Xiang 劉向 (77-6 A.C.). Ed de Kaltenmark 1953. 
Liu Zhaorui 劉昭瑞. 2001. «Cong kaogu cailiao kan daojiao toulong yijian lun toulong yi de jiyuan 從考古材料看道教投龍儀一兼論投龍儀 的起源》. Daojia yu daojiao 道家與道教: Daojiao juan 道教卷,

Guangzhou, Guangdong renmin chubanshe : 475-501.

Lüshi chunqiu 呂氏春秋. Lü Buwei 呂不韋 (m.235 A.C.) et autres. Ed. de Chen Qiyou 陳奇猷, Lüshi chunqiu jiaoshi 呂氏春秋校釋, Shanghai : Xuelin, 1984.

Miyakawa Hisayuki. 1979. «Local Cults around Mount Lu at the time of Sun En's Rebellion ». Facets of Taoism : Essays in Chinese Religion, Holmes Welch \& Anna Seidel ed., New Haven, Yale University Press, $1979: 83-101$.

Needham, J. et al. 1976. Science and Civilisation in China, vol. 5 :

Chemistry and Chemical Technology, part 2 : Spagyrical Discovery and Invention : Magisteries of Gold and Immortality, Cambridge, Cambridge Univ. Press.

Niida Noboru 仁井田梸. 1937. TôSô hôritsu bunsho no kenkyû 唐宋法律文 書の研究. Tôhôbunka gakuin, Tôkyô kenkyûsho.

Ôfuchi Ninji. 1974. « On Ku Ling-pao-ching ». Acta asiatica, 27 : 33-56.

Pregadio, F. 1991. " The Book of the Nine Elixirs and Its Tradition ». Chûgoku kodai kagakushi ron, édité par Yamada Keiji and Tanaka Tan, Kyoto, Jinbun kagaku kenkyûjo, 1991 : 543-639.

Rao Zongyi 饒宗頣. 1989. Gu'an wenlu 固庵文錄. Taipei, Xinwenfeng chuban gongsi.

Robinet, I. 1984. La révélation du Shangqing dans l'histoire du taoïsme. Tome second. Paris, Ecole Française d'Extrême-Orient.

Robinet, I. 1996. Comprendre le Tao. Paris, Bayard. Réédition : Paris, Albin Michel, 2002.

des Rotours, R. 1952. «Les insignes en deux parties (fou 符) sous la dynastie des Tang (618-907) ». T'oung Pao, 41 (1952) : 1-148.

Schipper, K. 1991. «Reihô kagi no tenkai 靈寶科儀の展開》. Nihon Chûgoku no shûkyô bunka no kenkyû 日本中國の宗教文化の研究, Tokyo, Hirakawa shupansha : 219-231.

Shanhaijing 山海經. Textes des dates variées du ca. $4^{\mathrm{e}}$ siècle A.C. au ca. $1^{\mathrm{er}}$ siècle P.C. Ed. de Yuan Ke 袁珂, Shanhaijing jiaozhu 山海經校注, Shanghai : Shanghai guji chubanshe, 1980.

Shandongsheng bowuguan 山東省博物館 et Cangshanxian wenhua guan 蒼 山縣文化館. 1975. 《Shandong Cangshan Yuanjia yuannian huaxiang shimu 山東蒼山元嘉元年畫像石墓》. Kaogu, 1975/2:124-134.

Shanxisheng kaogu yanjiusuo 山西省考古研究所 et al. 1994. «Shanxi Xiaxian Wangcun Donghan Bihuamu 山西夏縣王村東漢壁畫墓 》. Wenwu, 1994/8 : 34-46.

Shavkunov, E.V. 1989. «Sulian Nikulayefuzike yizhi chutu de yuxing qingtong xinfu 蘇聯尼古拉耶夫斯克遺址出土的魚形青銅信符》. Beifang wenwu, 1991/1 : 102-103. Traduction chinoise de l'article originel en russe publié dans Sovestskaya arkheologiya, 1989/1 : 267-270. 
Stein, R.A. 1943. « Jardin en miniature d'extrême-orient ». Le Monde en petit, Paris : Idées et Recherches/Flammarion, 1987 : 13-118. Article originairement publié dans BEFEO, t. XLII, 1, Hanoi, 1943.

Stein, R.A. 1947. «Le Lin-yi 林邑》. Han-hiue, vol. 2, fascicules 1-3.

Stein, R.A. 1968. « Textes taoïstes relatifs à la transmission des livres révélés ». Annuaire du Collège de France, 68 (1967/1968) : 453-457.

Tai Jingnong 臺靜農. 1975. « Tang Minghuang Qingchengshan chi yu Nanyue gaowen 唐明皇青城山敕與南获告文》. Jingnong lunwen ji 靜 農論文集, Taipei, Lianqing chuban, 1989 : 317-324. L'article est originairement publié dans Shumu jikan 書目季刊 vol. 8, no.4 (1975/3).

Taiqingjing tianshi koujue 太清經天師口訣. Six Dynasties. Daozang (CT 883).

Taishang dongxuan lingbao chishu yujue miaojing 太上洞玄靈寶赤書玉訣 妙經. Avant $5^{\mathrm{e}}$ siècle. Daozang (CT 352).

Taishang dongxuan lingbao shoudu yi 太上洞玄靈寶授度儀. Lu Xiujing 陸修靜 (406-477). Daozang (CT 528)

Taishang dongxuan lingbao zongjian wen 太上洞玄靈寶衆簡文. Lu Xiujing (406-477). Daozang (CT 410).

Waley, A. tr. 1955. The Nine Songs : A Study of Shamanism in Ancient China, London: George Allen \& Unwin LTD.

Wang Sili 王思禮. 1958. «Shandong Feicheng Han huaxiang shimu diaocha 山東肥城漢畫像石墓調查》. Wenwu cankao ziliao 文物參考資料, 1958/4 : 34-36.

Wang Yucheng 王育成. 1994. «Ming Wudang shan jinlong yujian yu daojiao toulong 明当山金龙玉简与道教投龙 》. Shehui kexue zhanxian 社会科学战线, 1994/3:148-154.

Wu Hung. 1989. The Wu Liang Shrine. The Ideology of Early Chinese Pictorial Art. Stanford, Stanford University Press.

Wu Hung. 1994. «Beyond the 'Great Boundary' : Funerary Narrative in the Cangshan Tomb ». Boundaries in China. John Hay ed., London, Reaktion Books, 1994 : 81-104.

Yamada Toshiaki 山田利明. 1999. Rikuchô dôkyô girei no kenkŷu 六朝道教 儀禮の研究. Tokyo, Tôhô shoten.

Zaozhuangshi wenwu guanli weiyuanhui bangongshi 春莊市文物管理委員 會辦公室 et Zaozhuangshi bowuguan 奉莊市博物館. 1997. «Shandong zaozhuang xiaoshan xihan huaxiang shimu 山東春莊小山西漢畫像石 墓 》. Wenwu, 1997/12:34-43.

Zhang Zehong 張澤洪. 1996. «Daojiao zhaijiao yuanliu chuxi 道教齋醮源 流媰析》. Zongjiaoxue yanjiu 宗教學研究 1996/3 : 26-31.

Zhen'gao 真誥. Tao Hongjing 陶弘景 (456-536). éd. Daozang (CT 1016).

Zhou Xibo 周西波. 1999. «Dunhuang xiejuan P.2354 yu Tangdai daojiao toulong huodong 敦煌寫卷 P.2354 與唐代道教投龍活動》. Dunhuang xue 敦煌學 22, 1999/12:91-109. 
KIM Daeyeol

Doctorat (nouveau régime) in History of religions and religious anthropology, University of Paris-Sorbonne (Paris IV), December 2000 : «Le symbolisme de la force vitale en Chine ancienne: Modèles et significations dans l'alchimie taoïste opératoire (études des pratiques alchimiques du Baopuzi neipian)». Maître de conférence at the Institut National des Langues et Civilisations Orientales (Langues'O). Recent Publication : «Métallurgie et alchimie en Chine ancienne », Eurasie 12, La Forge et le forgeron, II. Le merveilleux métallurgique, Paris : l'Harmattan, 2003, p.155-189. 


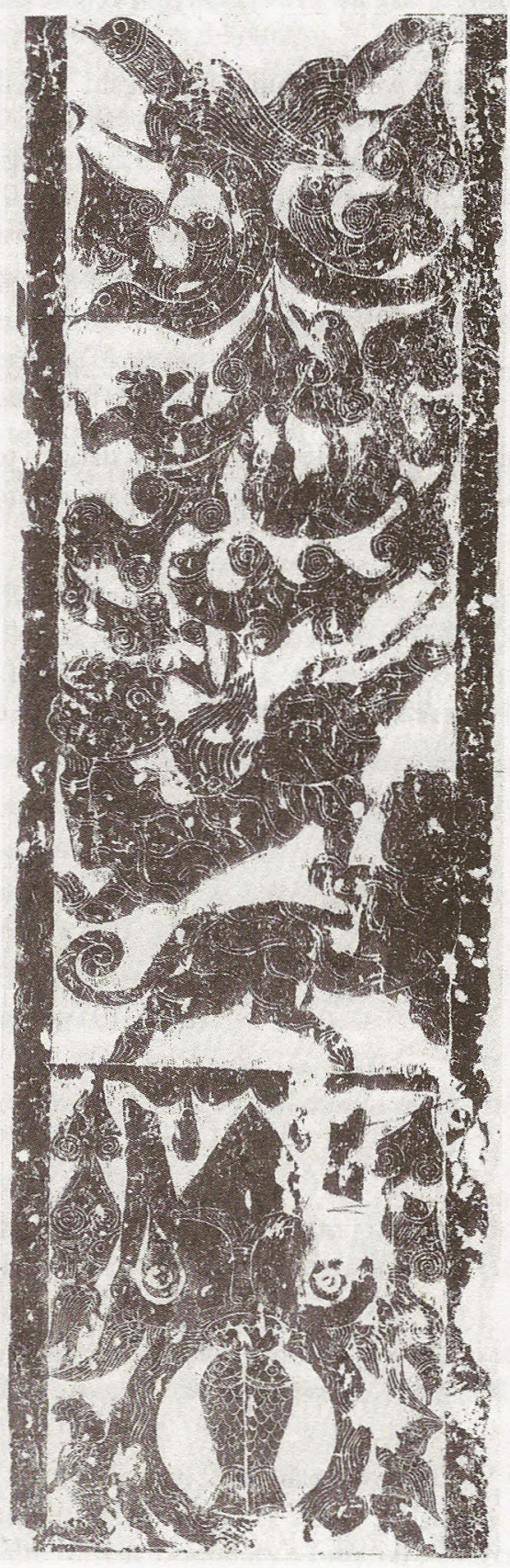

Fig. 1

Liaocheng diqu bowuguan 1989, p. 53 


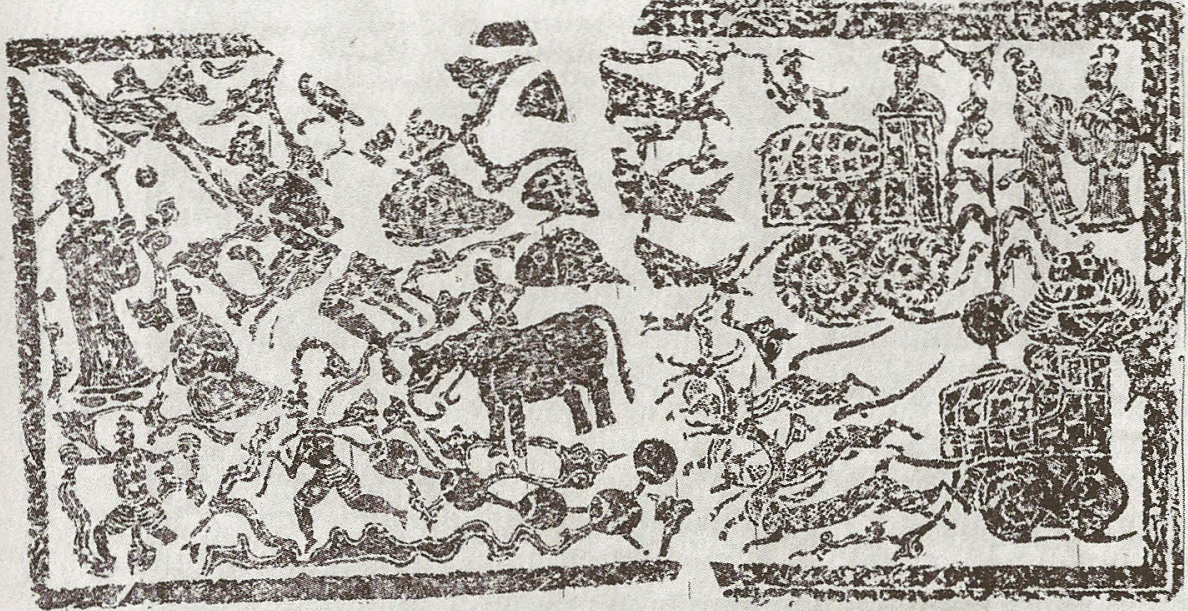

Fig. 2

Jiangsusheng wenwu guanli weiyuanhui 1959, fig. $\mathrm{n}^{\circ} 52$.

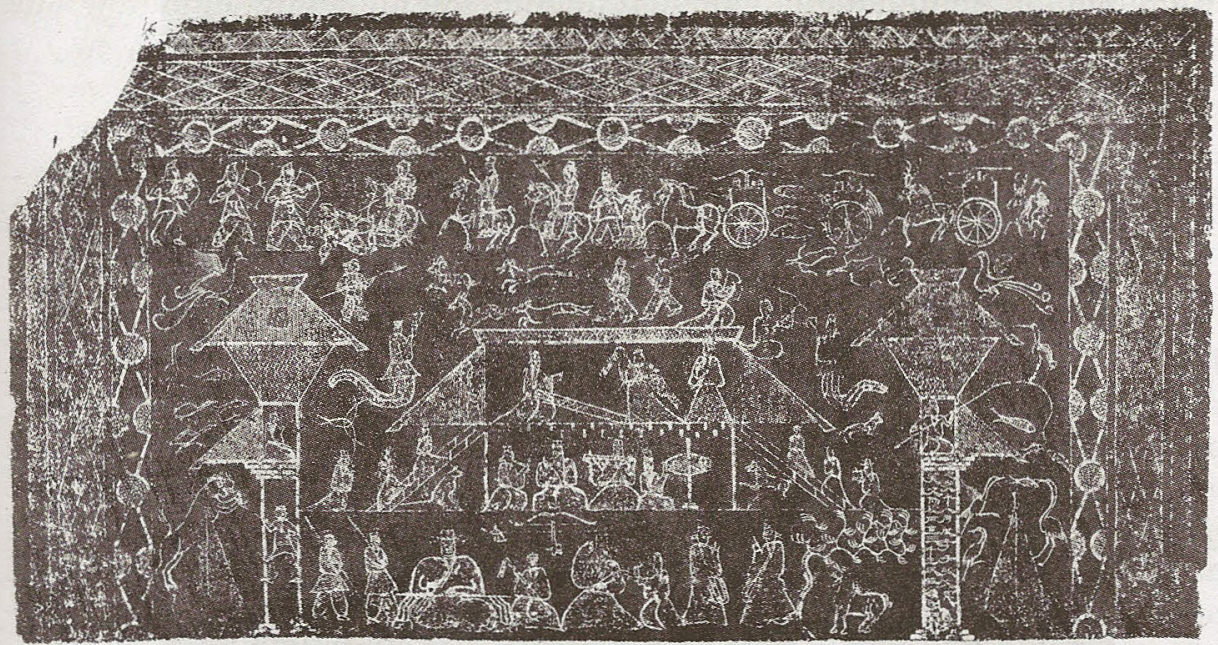

Fig. 3

Wang Sili 1958, p. 35. 


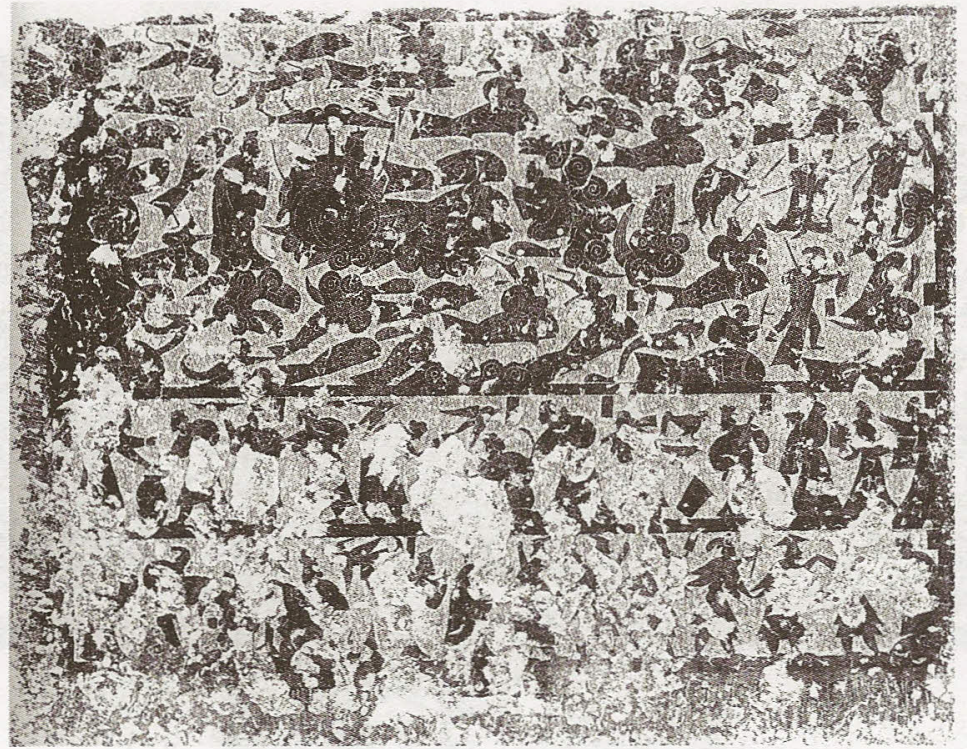

Fig. 4

Chavannes $1909, \mathrm{n}^{\circ} 130$.

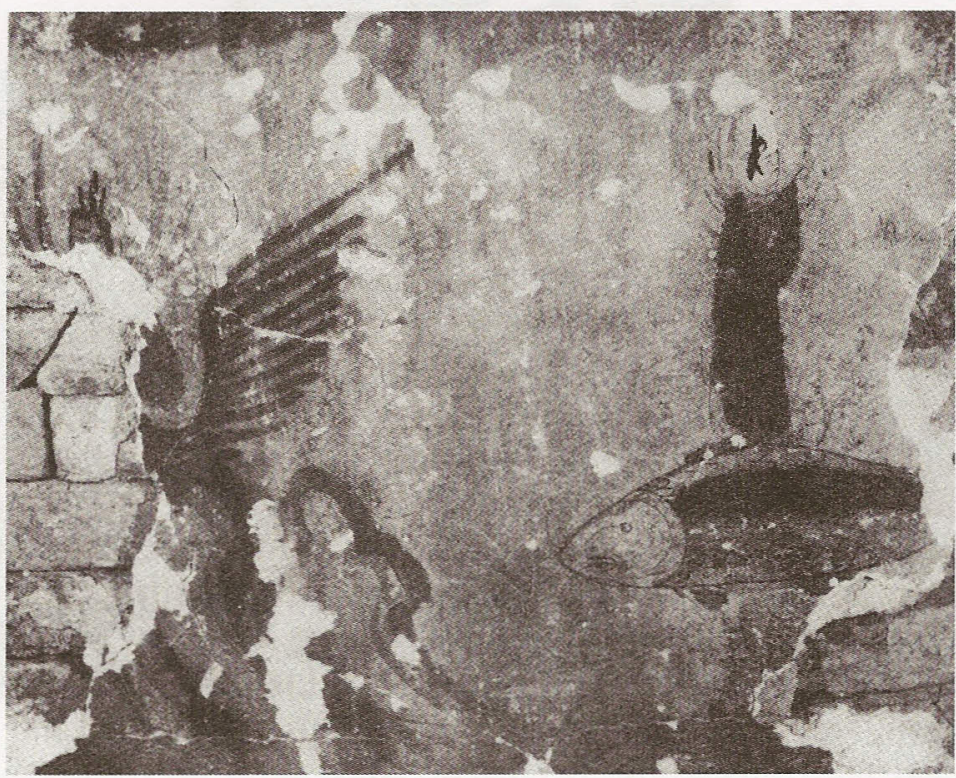

Fig. 5

Shanxisheng kaogu yanjiusuo et al. 1994, p. 36. 